\title{
Structure analysis of human Prion protein involved in Sporadic Fatal Insomnia
}

\author{
SHORT COMMUNICATION
}

Pardis Tabaee Damavandi

Queen Mary University of London, Mile End road, London, UK

p.tabaee@qmul.ac.uk

\begin{abstract}
Prion disorders are the root cause of Transmissible Spongiform Encephalopathies (TSE), a group of lethal diseases portrayed by progressive neurodegeneration and spongiosis. In recent years, researchers have come to understand that it is not the endogenous presence of Prions itself that causes neurodegeneration, but the amount of prion proteins that accumulates in the nervous tissue, leading them to exert neurotoxicity. More specifically, the cause of these disorders is mapped to several mutations that can bring the prion protein structure to a disordered permanent misfolded state. Our research is focused on Sporadic Fatal Insomnia (sFI), a rare TSE characterized by severe and chronic insomnia, leading to a life expectancy estimation of about two and a half years, from the onset of the first symptoms. The goal of this work was to analyze through computational studies the structure of the native human Prion Protein $\left(\mathrm{PrP}_{\mathrm{nat}}\right)$ and compare it with the toxic form (FI-Prion) which causes disease. Our findings show that the structure of the human mutant FI-Prion, responsible for Sporadic Fatal Insomnia is more flexible than the native human form $\mathrm{PrP}_{\text {nat }}$. Specific regions of the mutant seem to fluctuate more freely than the corresponding loops in the native form. We also identified amino acids Tyr128 and Met129 to be the key residues playing a major role in the manifestation of the disease. Therefore, we've learnt that the FI-Prion is more flexible than $\operatorname{PrP}_{\text {nat. }}$ In addition, we also confirmed that sporadic fatal insomnia is undoubtedly an infectious disease.
\end{abstract}

Keywords Computational biology, Prion disease, Protein misfolding, Tyrosine, Fungi.

\section{INTRODUCTION}

Since the war era, it was known that pesticides $(1,2)$ and other nerve agents were able to cause neurotoxicity in humans and other species (3). Chemical or chemotherapeutic compounds weren't the only agents demonstrating toxicological effects, but other etiological organisms, including bacteria, parasites and neurotoxins present in poisons (produced by insects for example) were equally able to act as infectious agents, provoking cellular damage. Endogenous molecules, such as inflammatory cytokines similarly were reported to have some kind of role in sleep disorders, by reducing sleep and by up- or downregulating the sleep cycle (4).

Other studies infer that the inoculation of cysts in the brain, by parasites which originally accessed our organism via the intestinal epithelial cell barrier route, results in an excessive inflammatory response that can cause neuronal damage (5). This has led to other findings suggesting that the passage of the etiological agent causing Transmissible Spongiform Encephalopathies (TSE) from host to host takes place in the human intestinal epithelial cell barrier. Logically, this would be possible due to the low molecular weight of the prion protein $(\sim 35-36$ $\mathrm{kDa} ; 6)$.

Our hypothesis, based on computational sequence analysis studies, is the possibility that like cysts, even prions, may be a result of fungal hydrolysation in another host, and byproducts transported to other hosts by microbes. The ultimate passage from gut through the bloodstream may not require microbial action, but could be facilitated by the prion's own small size, and the trophic ability to reach and access the blood brain barrier (BBB) may be eased by mimesis (7). 
TSE have often also been classified as autoimmune diseases, not only for their inflammatory and degenerative 'quality'(8), but because they have been linked to the processes of neuroprotection and autoimmunity (9).

Likewise, Zhu theorised that the mechanism of conversion from the healthy Prion Protein (or $\mathrm{PrP}^{\mathrm{c}}$ ) into its pathogenic isoform, $\mathrm{PrP}^{\mathrm{sc}}$, may be mediated by antibodies targeting $\mathrm{PrP}^{\mathrm{sc}}$ itself (10). Analogous observations have also been made by Liberski (2008; 11).

Although there is not enough evidence on which microorganism is responsible for the mutation of prions, there have been studies suggesting that prion proteins are normally present as membrane glycoproteins, though, unlike other proteins, their mutated forms are able to survive in extreme conditions (12).

Several studies (13) also prove that it is possible to produce resistant prion isoforms $\mathrm{PrP}^{\text {res/sc }}$ (protease-resistant prion) and infect a healthy host. A few days post-inoculation, the host develops TSE. This indicates that mutant prions are undoubtedly infectious.

Late advances in Alzheimer's disease, where $\beta$-amyloid fibrils were considered 'responsible' for the illness, suggest that $\beta$-amyloid filaments become pathologically inactive when separated from the mutant prion protein. This signifies that, like Sporadic Fatal Insomnia, Alzheimer's Disease is a prion disease too.

As mentioned above, Liberski et al (2008) carried out laboratory experiments explaining how the biosynthesis of both the healthy $\left(\mathrm{PrP}^{\mathrm{c}}\right)$ and unhealthy $\left(\mathrm{PrP}^{\mathrm{TSE}}\right)$ prion proteins, encoded by gene PRNP, starts in the endoplasmic reticulum and ends with the expression of $\operatorname{PrP}^{\mathrm{c}}$ as a surface membrane glycoprotein. They also reveal that the metabolism consists in complete proteolytic degradation for the healthy form of the prion protein $\left(\mathrm{PrP}^{\mathrm{c}}\right)$, but not for the mutant one $\left(\mathrm{PrP}^{\mathrm{TSE}}\right)$. This leads to extracellular "free $\mathrm{PrP}^{\mathrm{TSE}}$ ". Because prion proteins lack of nucleic acids and massive vacuolation is present, he hypothesises that the mechanism of neuronal cell death in Fatal Familial Insomnia may be both apoptotic and autophagic (11).

Unlike ALD (amyotrophic lateral dystrophy), where unmyelinated vacuoles are deeper or superficial in the cortical layers but in specific locations, attenuation of white matter is not present. In fatal insomnia, neurodegeneration is depicted by a progressive softening of the thalamus to reach a "status spongius" in its final stage, and it almost resembles Polioencephalomalacia without the hydrocephalic features.

Recent studies, conducted by Chiesa (2016) demonstrate that sometimes the conversion of native prions into their mutant toxic isoforms is not necessary for the disease to take place. This new revelation is useful in understanding why neurodegeneration is heterogeneous. He also states that the reason why the mutant prion isoforms in FI are infectious is because they accumulate in front of the voltage-gated calcium channels (VGCC), preventing normal electrolytic transmission and leading to general neuronal transmission defects. The result is a cascade in which the cluttering of the channels by the mutant isoforms is disruptive to the healthy native prion functions, whose main role consists in conferring protection to the neurons (14).

A better analysis of human prion structures is therefore important in understanding Sporadic Fatal Insomnia (sFI) and will be discussed in the next section.

\section{BACKGROUND}

The name "Prion" which means "proteinaceous" and "infectious" was coined by Prusiner, who purified it by centrifugation, in 1998 (15). The nature of this membrane glycoprotein was initially mistaken for that of a DNA slow virus, later with a membrane fragment, and lastly with a protein. Hunter in 1972 (16) had identified the native prion protein as a membrane glycoprotein 
and recent studies (2008) also show that the native prion protein $\operatorname{PrP}^{c}$ hereby named "PrP $\mathrm{P}_{\text {nat }}$ " competes with neurotransmitter glutamate to bind the N-Methyl-D-Aspartate (NMDA) and the $\alpha$ amino-3-hydroxy-5-methyl-4-isoxazolepropionic acid (AMPA) receptors (17).

Prions are a family of proteins that are very heterogeneous. Although they are encoded by the same gene, PRNP, located on chromosome 20 in man, and derive from the same precursor molecule made of 253 amino acids, many conformers have been found. These conformers are linked to different mutations (Table 1), which cause different neurodegenerative disorders and symptoms. For years this has been thought to be caused by an improper folding.

More specifically, the native human prion precursor protein $\mathrm{PrP}_{\text {nat }}$ incorporates a flexible $\mathrm{N}$-terminal region, which is made of 22 residues cleaved in the Endoplasmic Reticulum, a globular hydrophobic middle region, and a C-terminal tail, which consists of 23 residues, also cleaved by glycosylation. A further glycosylation occurs in the Golgi Apparatus and once the mature prion protein is incorporated on the cell surface, further glycosylation may or may not occur (18).

Studies have shown that 7.2 is the optimal $\mathrm{pH}$ for healthy prions, whereas below this $\mathrm{pH}$, conversion from the healthy mature prion to the protease resistant isoform happens (19). Unfortunately, prion unfolding occurs initially as a two-stage process in a $\mathrm{pH}$ range of 7.0-5.0, followed by a three-stage unfolding further below that range. The intermediate conformer observed during the three-stage unfolding process is thermodynamically favoured. Unlike the other stages the stable intermediate is not an $\alpha$-helical conformer, but a $\beta$-sheet conformer. This is in agreement with the unfolding tendencies of other proteins.

In reality, Fatal Insomnia can be classified into Fatal Familial Insomnia and Sporadic Fatal Insomnia. The clinical features of the two disorders are similar, where both are characterised by the same point mutation at codon 129 , translated into a methionine-methionine homozygosity in the prion protein. On each alleles, this methionine replaces the original Val129 featured in the native healthy prion $\left(\mathrm{PrP}_{\text {nat }}\right)$.

The difference between the two disorders lies in another polymorphism at codon 178 where an aspartic acid (Asp178) is substituted with an asparagine (Asn178). This D178N mutation is found in people who suffer from the familial form of the disease (FFI), but not in those who are affected by its sporadic form (sFI; 20). For simplicity, the standard mutated prion which contains the Met129 in place of the valine residue, will be called "FI-Prion"; this prion has been the object of our analysis and computational studies. The structure of the FI-Prion involves a globular domain, extending between Leu125 to Arg228, an N-terminal flexible domain and a C-terminal region. Both the $\mathrm{N}$ - and $\mathrm{C}$-terminal extremities are proteolytically cleaved.

\section{SUMMARY OF OUR FINDINGS}

\section{PRION AND FI-PRION STRUCTURE ANALYSIS RESULTS}

To examine the prion structure, Lee et al (2010) co-crystallised seven PrP molecules. They also highlighted that of these, the wild-type un-mutated $\operatorname{PrP}_{\text {nat }}$ crystallised as closed monomers, whereas the mutants crystallised as unswapped dimers. Interestingly, the FI-prion structure was made available ( $\mathrm{pdb}$ code $3 \mathrm{HES}$ ) and the native unmutated $\mathrm{PrP}_{\text {nat }}$ prion ( $\mathrm{pdb}$ code $3 \mathrm{HAK}$ ) was also deposited on the Protein Data Bank.

On a side note, the structures of $\mathrm{D}_{1}(\mathrm{PDB}$ code $1 \mathrm{OZ5}), \mathrm{D}_{2}(\mathrm{pdb}$ code $5 \mathrm{AER})$ and $\mathrm{D}_{3}(\mathrm{pdb}$ code 3PBL) dopamine receptors were also released in 2003, 2015 and 2010 respectively (Figure 1).

As reported in previous studies, the prion globular domain is made of three $\alpha$-helices (21) and two $\beta$-sheets. More specifically, $\alpha 1$, the shortest helix, includes residues Asp144 to His155, $\alpha 2$, amino 
acids Gln172 to Lys 194 and $\alpha 3$, residues Glu200 to Arg228 making it the longest helix. Although the two $\beta$-sheets are barely visible in the wild-type prion $\operatorname{PrP}_{\text {nat }}$, they comprise residues Tyr162163, Ala133 and Leu130. Accordingly, we noted that the FI-Prion (FI-PrP ${ }_{129}$ ) also displays two explicit $\beta$-sheet strands, $\beta 1$ and $\beta 2$. $\beta 1$ is made of the especially rigid Tyr162, Val161 and Gln 160 . $\beta 2$, on the other hand, mainly consists of the particularly flexible mutant amino acid Met129, of Leu130, Gly131 and Ser132.

Prion proteins only exhibit one disulphide bridge Cys179-Cys214 separating the two longest helices (Figure 2). This cysteinic pons has been associated with amyloid fibril formation (22).

In recent years, Lee et al (2010) reported the conformation variability of loop Arg164Ser170 in the PrP (prion protein) structures, where Arg164 binds Asp167 in a salt bridge, and Tyr128 forms a hydrogen bond with the mutant Asn178 (FFI-prion). They also observed that Tyr169 loses a hydrogen bond with Asn178, but that the repulsion swings it towards Glu168 leading to the formation of a hydrogen bond.

Other residues that have demonstrated to have catalytic activity are Arg164-Tyr169, Tyr218, Glu222 and Gln212.

We observed that the active site of FI-Prion consists of mainly amino acids Tyr162, Tyr163 belonging to the $\beta$-sheet motif, of residue Met129 and of residues His177-His187 in the $\alpha 2$ helix, where Gln186, Ile182, Asp178, His177 seem prominent. Another essential amino acid is Tyr128, which may be indirectly coordinated by both Asp178 and Met129 via hydrogen bonds (Figure 3). The key residue that may play a major role in prion folding seems to be Met129, accompanied by its hydrophobic neighbours Tyr128, Leu130 and Tyr163. Per contra, other methionine residues surrounded by positively charged amino acids, i.e. lysine or arginine, confer $\alpha$-helicity to the prion.

Our further findings also suggest that most tyrosine residues of FI-Prion are quite flexible. We also compared the glutamate NMDA receptor GluN1A/GluN2B, released in 2014 (pdb code 4PE5) and AMPA ionotropic receptor, released in 2009 (pdb code 3KG2) of Rattus Norvegicus, and found that they display partial sequence homology with the human FI-Prion (Figure 4). Both receptors expose a tyrosine in position 128, but the NMDA sequence also includes key residues Tyr823-Gly826 comparable with Tyr128-Gly140 of FI-Prion, and Gly44-His53 comparable with Glu168-His177 found in FI-PrP 129 . The AMPA receptor also shares residues corresponding with the $\alpha 1$ and N-terminal regions of human FI-Prion (FI-PrP 129 ).

Nonetheless, Tyr218 can be found in the human NMDA receptor (pdb code 5KCJ), however, like human AMPA (pdb code 5H8S), most of the analogies with FI-PrP ${ }_{129}$ are only 'superficial'.

A further examination of the dopamine receptors D3 (DRD3) and D2 (DRD2) revealed that they also include a Tyrosine in position 129, whereas D1 (DRD1) seems to miss it. Dopamine D3 receptors share a homology with the longest helix of FI-Prion, $\alpha 3$. This can be unmasked by superimposing FI-Prion residues Thr199-Ala224 on reciprocal Val107-Val132 in DRD3. Hither could also explain why the dopamine agonist Piribedil is effective against Parkinson's Disease (23).

\section{NATIVE PRION AND FI-PRION MOLECULAR DYNAMICS STUDIES}

To better understand the differences in flexibility and therefore folding behaviour between the native prion protein and its mutated form involved in sporadic fatal insomnia (sFI), two molecular dynamics (MD) studies were considered. The MD simulations were actually run for each structure individually and for long simulations in water. The flexibility was investigated via 
$R M S F$ (root mean square fluctuations) and $B-F A C T O R$ methods, in particular, the $R M S F$ analysis with the two structures, aligned and compared is displayed in "Figure 6".

The RMSF analysis was conducted on backbone atoms. The results, in "Figure 6", show that the native prion is overall more rigid than the FI-Prion. To understand why this happens in more detail, the FI-Prion was selected for a $R M S F$ analysis per residue. The analysis describes the mobility of each residue during the simulation, where residues of interest were selected. An analysis of the whole $R M S F$ indicated that the most flexible amino acid is residue 219 , the mutation of which is involved in CJD (Table 1). As expected, the RMSF per residue over time also suggests that most tyrosine and phenylalanine residues of FI-Prion are quite flexible. Tyr128 also showed a high degree of flexibility. It can also be observed that the mutation of Val129 to Met129, affects the flexibility of other residues, in particular that of Asp178.

Furthermore, the substitution of valine with the methionine makes Tyr128 more flexible and indirectly causes it to point towards the water (Figure 7). On the contrary, when valine is present, Tyr128 acts as a "gate-door" in combination with Tyr162 by forming hydrogen bonds, and this "distant" gate is quite stable.

Lastly, the RMSF MD simulation results were converted into B-FACTORS which were then correlated to their experimental counterparts (Figure 8). These results confirmed that the disease is infectious and provided groundwork for further investigation and preliminary design of plausible therapeutic agents (30-32).

A short analysis for crystal points was done prior to finalization, eliminating the risk of false positives.

Acknowledgments. I would like to thank my supervisors, Profs Martin Dove and Richard Pickersgill, in assessing whether my work made sense (crystal point suggestion). I would also like to thank my tutors Profs Asa Barber and Rainer Klages for support during the drawing up phase of the work, Prof Philip Camp for initial moral support, with his extensive expertise in the field of computational chemistry.

Ethical disclosure. The author declares no conflict of interest.

I would like to dedicate this work primarily to the people suffering from Sporadic Fatal Insomnia or people in urgent need. This work is thus not available for reproduction without consent as the intellectual property of the content lies solely within the author and the journal or preprint journal (agrixiv-osf) publishing it, nor any authorization for publication of its content has presently been provided elsewhere. 


\section{References and Notes:}

1. L. R. Moddy et al., "Comparative prion disease gene expression profiling using the prion disease mimetic, cuprizone." Prion, 3(2), 99-109 (2009).

2. D. R. Brown et al., "The cellular prion protein binds copper in vivo." Nature, 390(6661), 684687 (1997).

3. P. B. Foley et al., "Encephalitis lethargica and the influenza virus. II. The influenza pandemic of 1918/19 and encephalitis lethargica: epidemiology and symptoms." J Neural Transm., 116(10), 1295-1308 (2009).

4. C. Carnovale et al., "On the association between human papillomavirus vaccine and sleep disorders: evaluation based on vaccine adverse events reporting systems." J Neural Scienc., (in press).

5. H. H. Garcia et al., "Taenia solium cysticercosis." Lancet, 362(9383), 547-556 (2003).

6. R. S. Mishra et al., "Protease-Resistant Human Prion Protein and Ferritin Are Cotransported across Caco-2 Epithelial Cells: Implications for Species Barrier in Prion Uptake from the Intestine" $J$ neurosci, 24(50), 11280-11290 (2004).

7. A. Nesler et al., "A complex protein derivative acts as biogenic elicitor of grapevine resistance against powdery mildew under field conditions" Front Plant Sci., 6, 715 (2015).

8. Q. Shi et al., "Brain microglia were activated in sporadic CJD but almost unchanged in fatal familial insomnia and G114V genetic CJD." Virol J, 10, 216 (2013)

9. S. K. Williams et al., "Neuroprotective effects of the cellular prion protein in autoimmune optic neuritis. The American journal of pathology" Am J Pathol., 178(6), 2823-2831 (2011).

10. B. T. Zhu et al.," Human and Animal Spongiform Encephalopathies Are the Result of Chronic Autoimmune Attack in the CNS: A Novel Medical Theory Supported by Overwhelming Experimental Evidence" Histol Histopathol., 20(2), 575-592 (2005).

11. P. P. Liberski et al., "Cell death and autophagy in prion diseases (transmissible spongiform encephalopathies" Folia Neuropathol., 46(1), 1-25 (2008).

12. D. M. Taylor et al., "Inactivation of prions by physical and chemical means." J Hosp. Infect., 43, S69-76 (1999).

13. J. I. Kim, "Mammalian Prions Generated from Bacterially Expressed Prion Protein in the Absence of Any Mammalian Cofactors" J Biol Chem., 285(19), 14083-14087 (2010).

14. R. Chiesa et al.," Transgenic mice recapitulate the phenotypic heterogeneity of genetic prion diseases without developing prion infectivity: Role of intracellular PrP retention in neurotoxicity" Prion, 10(2), 93-102 (2016).

15. S. B. Prusiner, "Prions" Proc Natl Acad Sci U S A, 95(23), 13363-13383 (1998).

16. G. D. Hunter, “Scrapie: A Prototype Slow Infection” J Infect Dis., 125, 427-440 (1972).

17. H. Khosravani et al., "Cellular Prion Protein Null Mice Display Normal AMPA Receptor Mediated Long Term Depression" Prion, 2(2), 48-50 (2008).

18. D. Ciric et al., "Biochemical insight into the prion protein family." Front Cell Dev Biol., 3, 5 (2015).

19. W. Swietnicki et al., "Familial Mutations and the Thermodynamic Stability of the Recombinant Human Prion Protein”J Biol Chem., 272, 27517-27520 (1997).

20. P. Cortelli et al., "Pre-symptomatic diagnosis in fatal familial insomnia: serial neurophysiological and ${ }^{18}$ FDG-PET studies", Brain, 239(Pt 3), 668-675 (2006).

21. S. Lee et al., "Conformational diversity in prion protein variants influences intermolecular beta-sheet formation." EMBO J., 29(1), 251-262 (2010). 
22. G. S. Jackson et al., "Reversible Conversion of Monomeric Human Prion Protein Between Native and Fibrilogenic Conformations" Science, 283(5409), 1935-1937 (1999).

23. P. Jenner, "Parkinson's disease: pathological mechanisms and actions of piribedil." J Neurol, 239, S2-8 (1992).

24. A. Plate et al., "Atypical parkinsonism due to a D202N Gerstmann-Sträussler-Scheinker prion protein mutation: first in vivo diagnosed case." Mov Disord., 28(2), 241-244 (2013).

25. P. S. Panegyres, "Codon 200 mutation of the prion gene: genotype-phenotype correlations." $J$ Neurol., 259(12), 2579-2584 (2012).

26. B. Sikorska et al., "Creutzfeldt-Jakob Disease" Subcell Biochem., 65, 457-496 (2012).

27. J. A. Mastrianni et al., "The genetics of prion diseases." Gen Med., 12, 187-195 (2010).

28. R. Medori et al., "Codon 178 Mutation and Codon 129 Polymorphism" Am J Hum Genet., 53(4), 822-827 (1993).

29. A. Kuzmanic et al., "Determination of Ensemble-Average Pairwise Root Mean-Square Deviation from Experimental B-Factors" Biophys J, 98(5), 861-871 (2010).

30. P. Tabaee Damavandi et al., "A review of drug therapy for sporadic fatal insomnia" (2017), Prion 1(5) 293-299.

31. P. Tabaee Damavandi et al., Master's Thesis. "Computational studies of falcipain-2 irreversible peptidomimetic inhibitors", (2010).

32. P. Tabaee Damavandi et al., Doctoral Thesis. "Novel applications of vibrational dynamics in the study of protein misfolding and neurodegenerative disorders", (2016). 
Figures

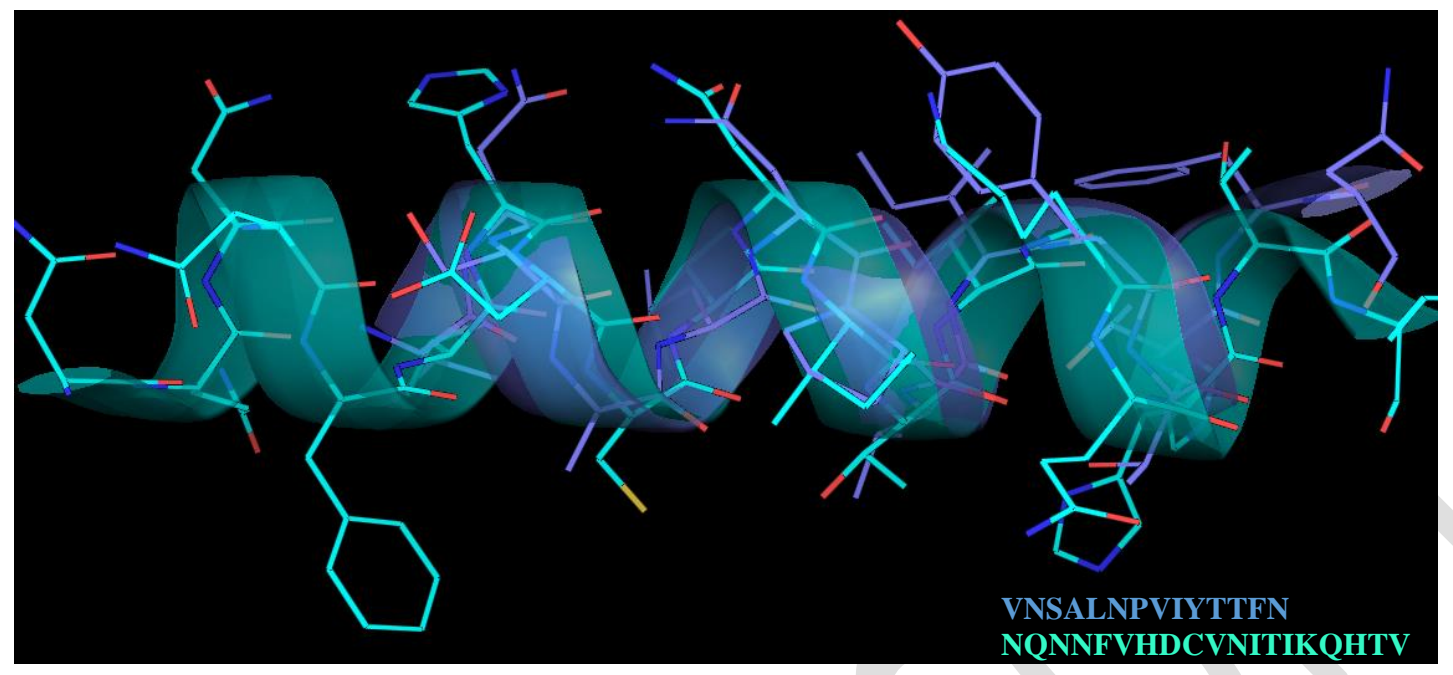

Fig 1. The figure shows the superposition of residues 374-386 of dopamine receptor DRD3 (dark blue) and the $\alpha 3$ helix (residues 171-186) of human prion protein $\mathrm{PrP}_{\text {nat }}$ (light blue).

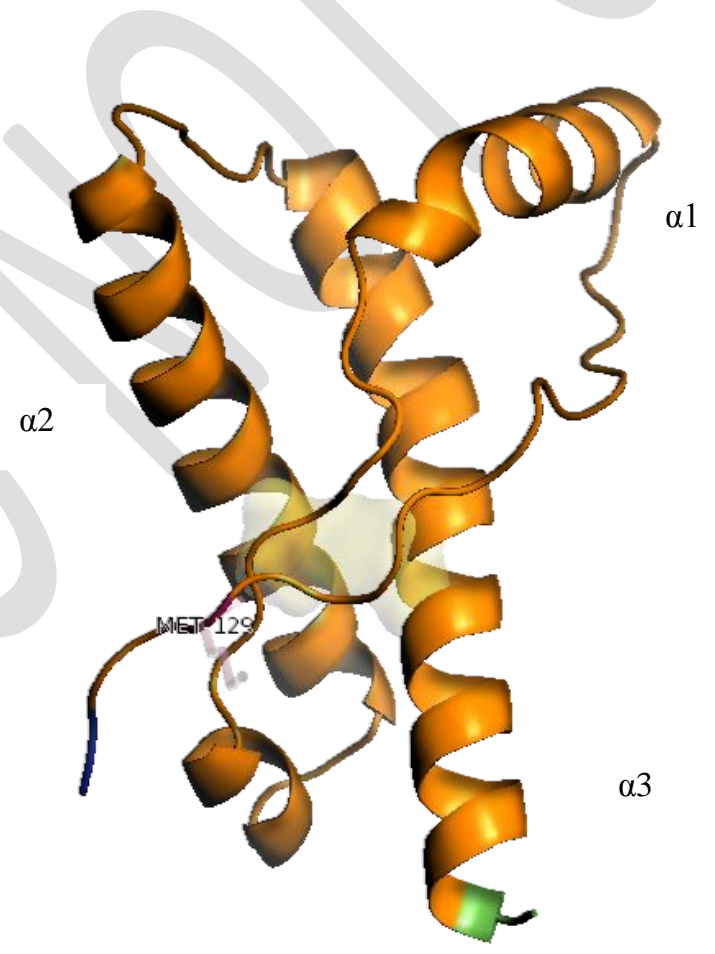

Fig. 2. The figure exhibits the Native Prion $\operatorname{PrP}_{\text {nat }}$ crystal structure. Highlighted are the residues extending towards the $\mathrm{N}$ terminus (blue) and the $\mathrm{C}$ terminus (green). The $\mathrm{N}$ terminal amino acid here reported is residue 125 . The nearby glycine 127 has shown to further stabilise prion folding, when it replaced a valine. The only disulphide bridge holding the protein together is shown in 
yellow. The $\alpha$ helices are also depicted, where $\alpha 1$ is the smallest helix (top right), subject to mutations seen in AD, $\alpha 2$ is the medium helix (on the left), of interest for FFI and CJD and $\alpha 3$ is the longest chain which extends to the $\mathrm{C}$ terminal region (on the right), mutations of which have been described in cases of PD and GSS [39-43]. The picture was obtained with Pymol.

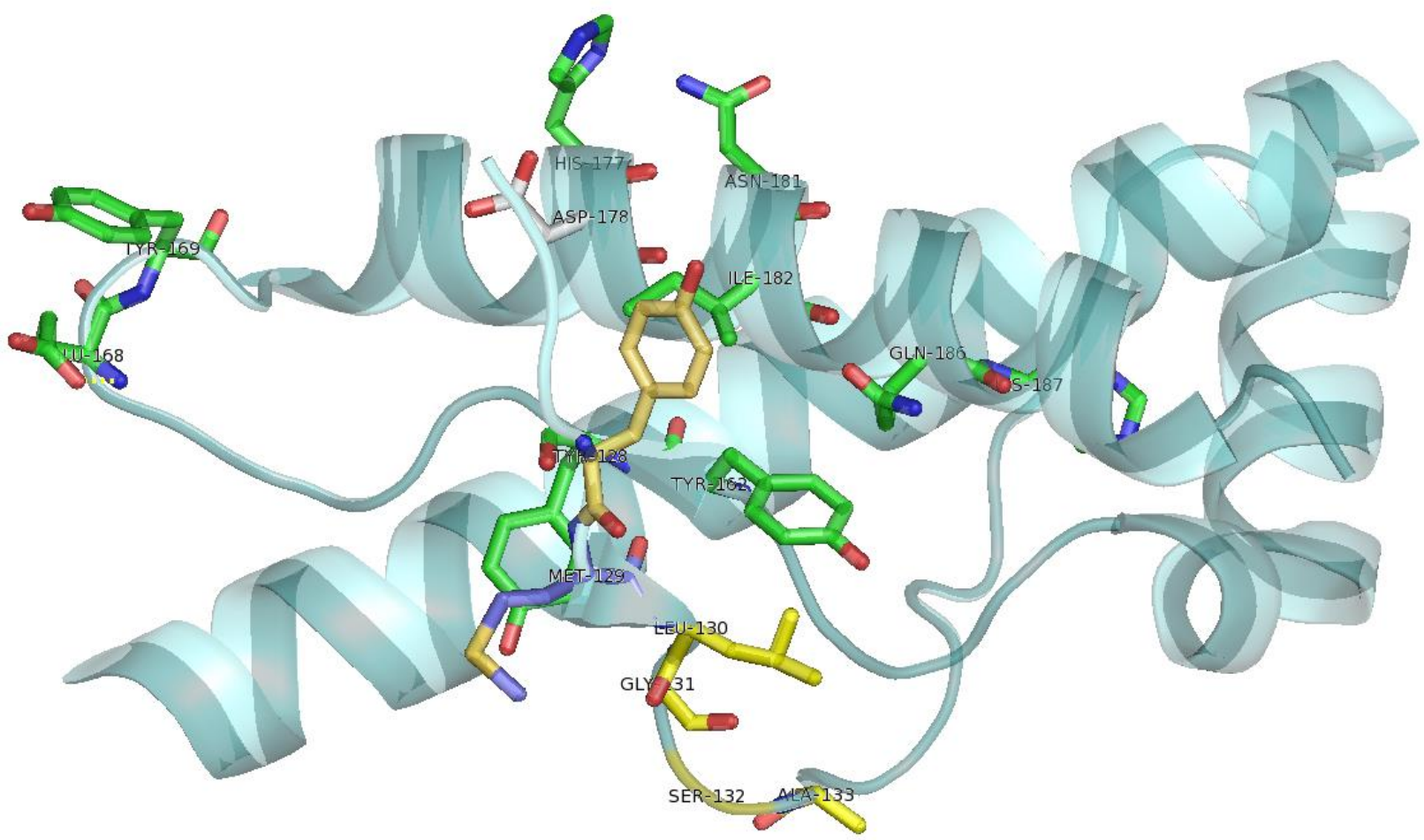

Fig. 3. The figure shows the key residues (green) obtained from the protein crystallographic structure of the human prion protein with the methionine 129 mutation (FI-prion). Met129 (in blue), Tyr128 (pale orange) and Asp 178 (gray) are therefore highlighted. Leu130-Ala133 residues 
(yellow) may have a role in the $\beta$-sheet formation, especially in the presence of Met129. The picture was obtained by using Pymol.

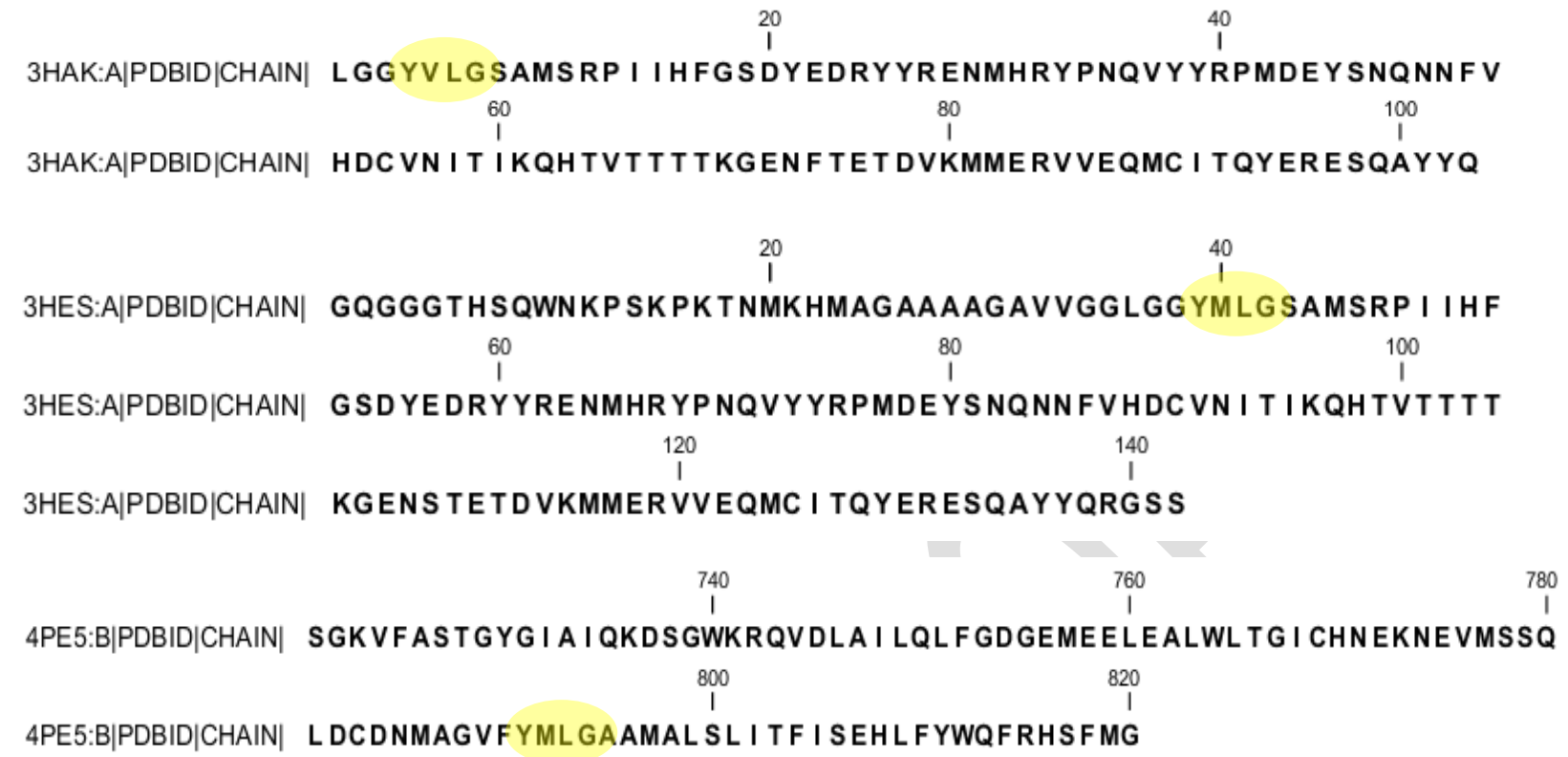

Fig. 4. The figure shows the sequencing of the healthy Prion protein (top), the unhealthy FI-Prion (middle) and the murine GluN2B NMDA receptor ion channel (bottom). In yellow are highlighted key sequences and the mutation $\mathrm{V}$ to $\mathrm{M}$. The presence of the same sequence which is responsible for the incorrect folding of the mutated prion (middle) compared to the native unmutated prion 
(top sequence) in Rattus Norvegicus (bottom) may lead to the conclusion that the possibility of passage from a host to another may not be excluded and that Prion disorders are infectious.

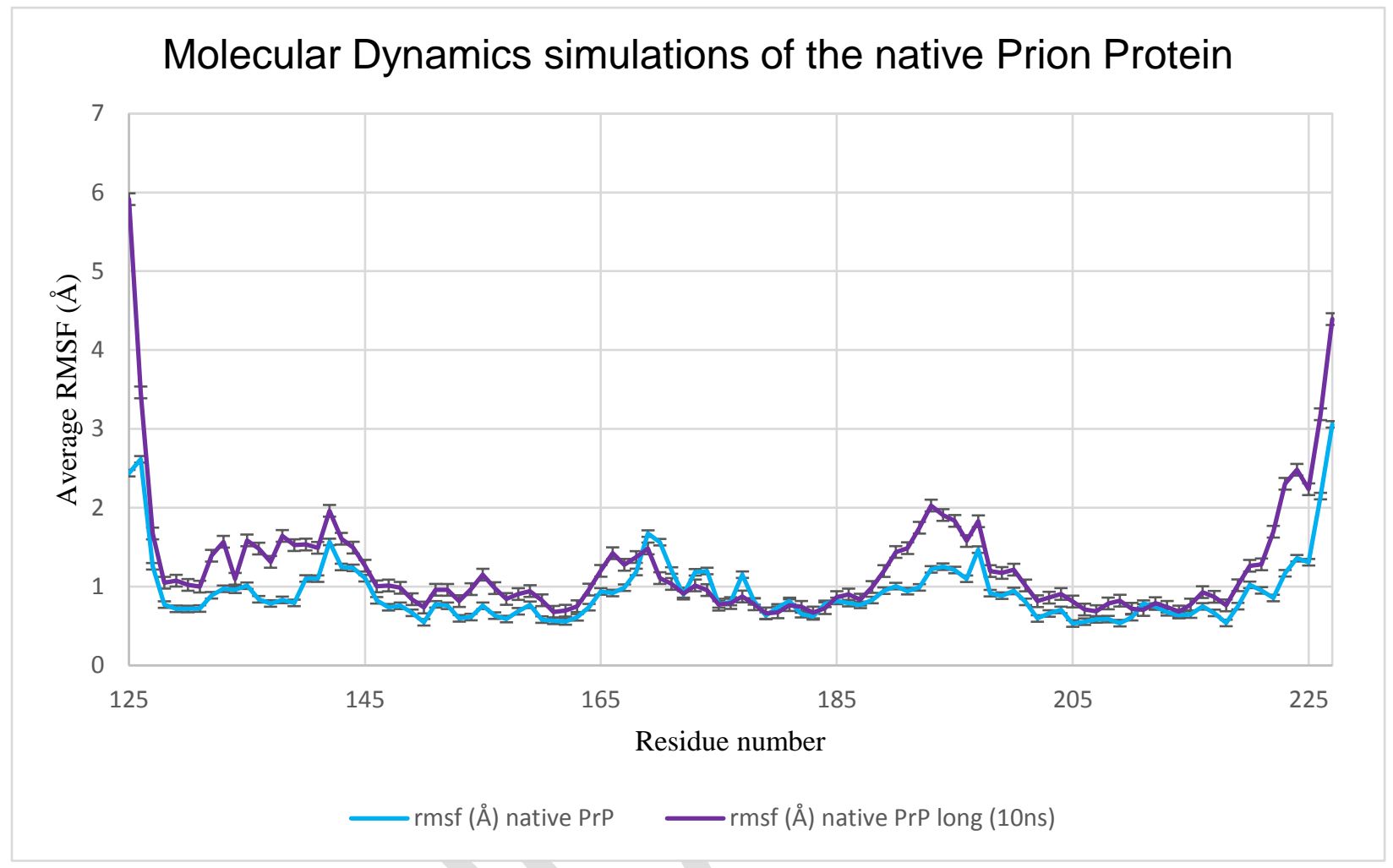

Fig. 5. The figure shows the backbone $\mathrm{C} \alpha$ root mean square fluctuation (RMSF) per residue for average structures of the native prion protein after short (light blue) and long (violet) Molecular 
Dynamics simulations. This picture shows that the RMSF can be used to understand the flexibility of residues.

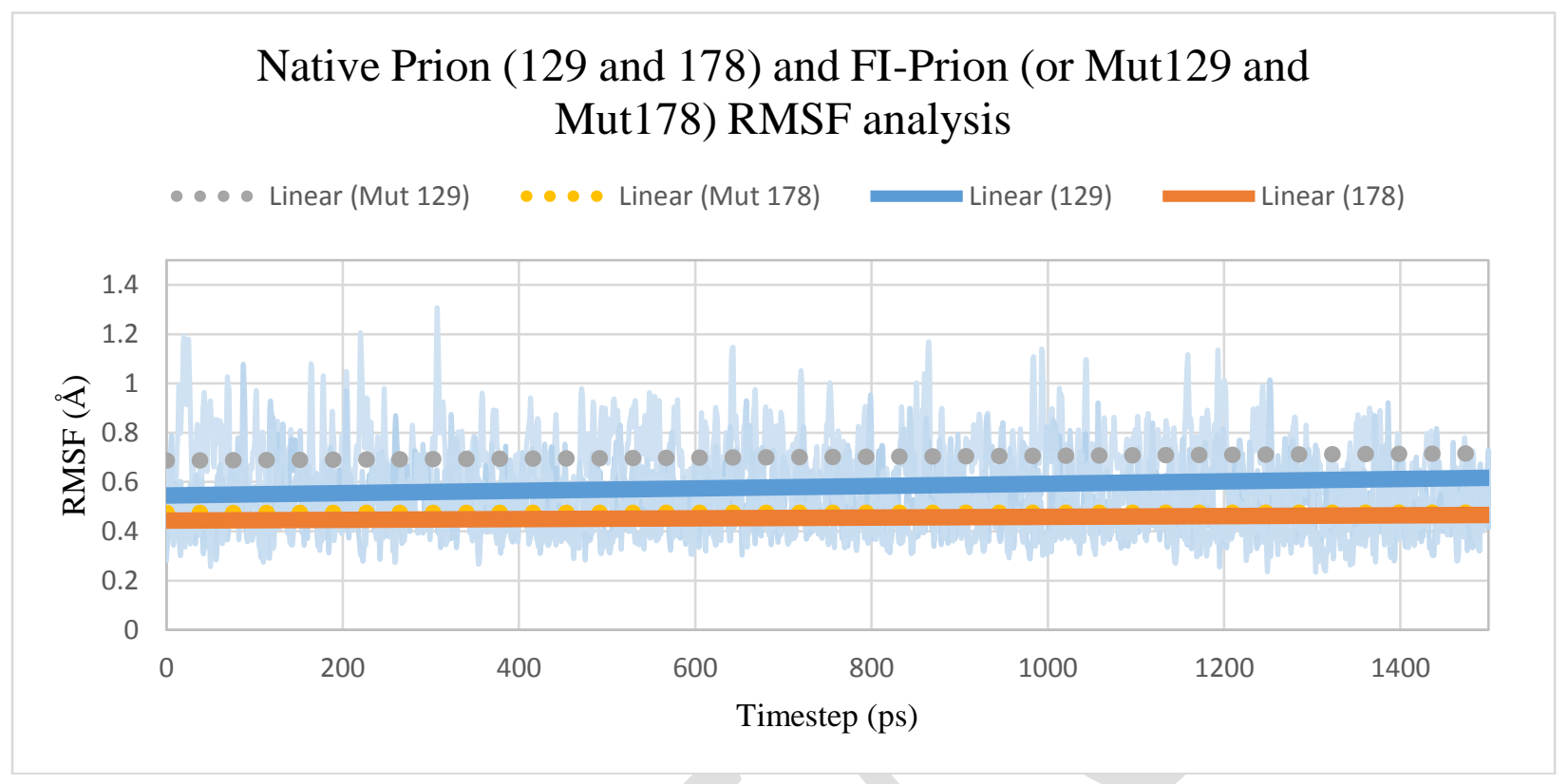

Fig. 6. The figure shows the backbone $\mathrm{C} \alpha$ root mean square fluctuation (RMSF) per residue over timestep of the native prion protein (squares) and the mutant FI-Prion (circles) after $1.5 \mathrm{~ns}$ of Molecular Dynamics simulation at high temperature $(364 \mathrm{~K})$. The only mutated amino acid in the mutant form is residue Met129 (yellow and gray), whereas the native prion protein contains valine Val129 at the same site (blue and orange). The squares and circles indicate the average flexibility, or residue mobility, of the structures. Overall, the native prion seems more stable and "rigid" than the FI-prion which holds the mutation and causes disease. The fluctuations show that the flexibility of Met129 influences the flexibility of other residues, in particular of Asp178. This can be seen prominently through the higher average values visible in the main residue Val129 of the native prion protein (blue squares) as opposed to the FI-prion (gray circles) containing the methionine in 
the same position, Met129. The V129M mutation was performed on the native prion protein original crystallographic structure (3HAK) and was achieved with NAMD2.2.

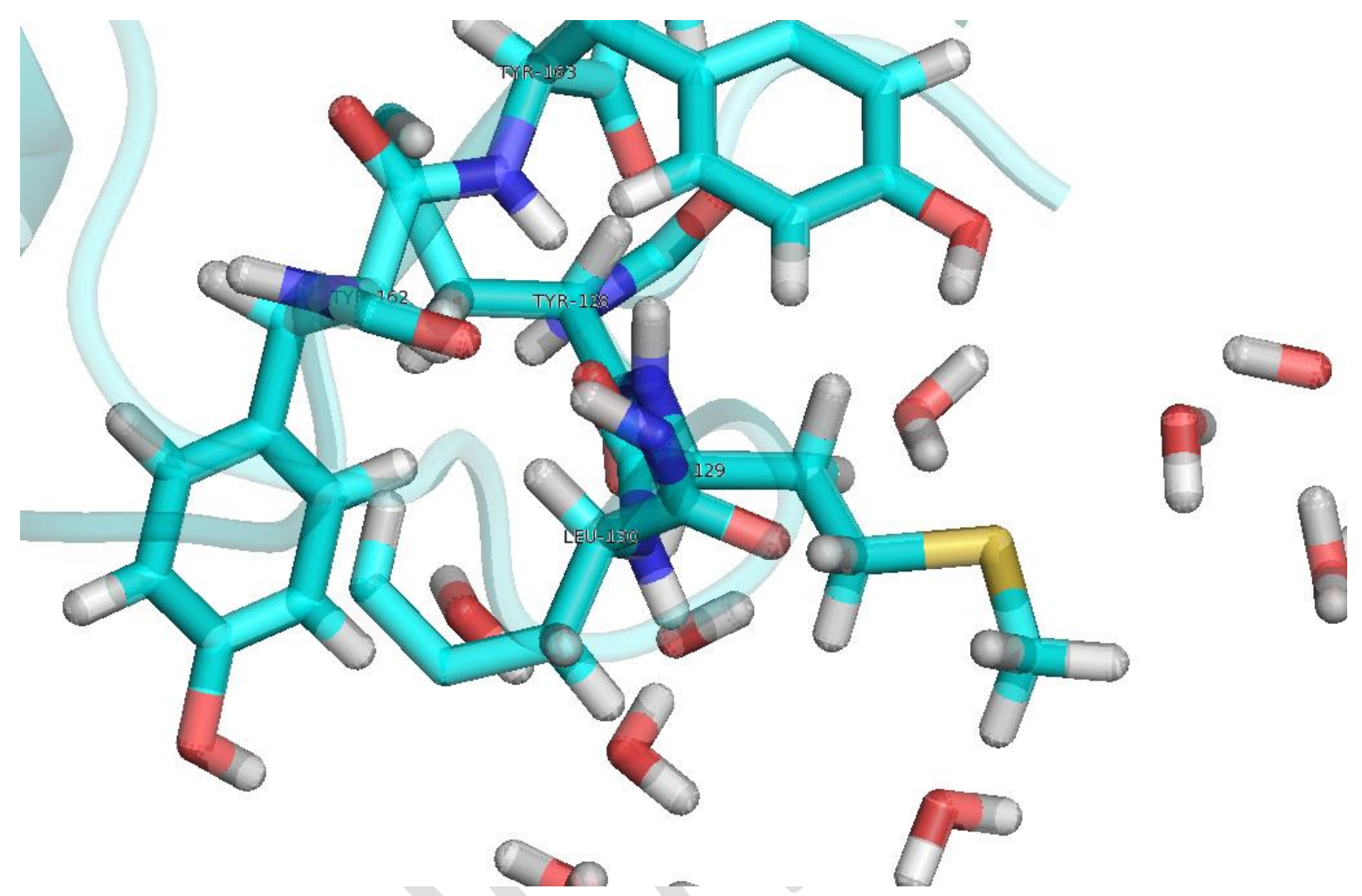

Fig. 7. The figure emphasises the $\mathrm{Met}_{129}-\mathrm{SCH}_{3}$ (yellow) flexible region of FI-Prion exposed to the surrounding solvent (water) after molecular dynamics simulation. No inter-molecular interactions are present, destabilizing the mutated residue and contributing to its highly flexible free 
movements. The molecular dynamics simulations also demonstrate that initially Asp178 is stabilized by the presence of nearby Tyr128.

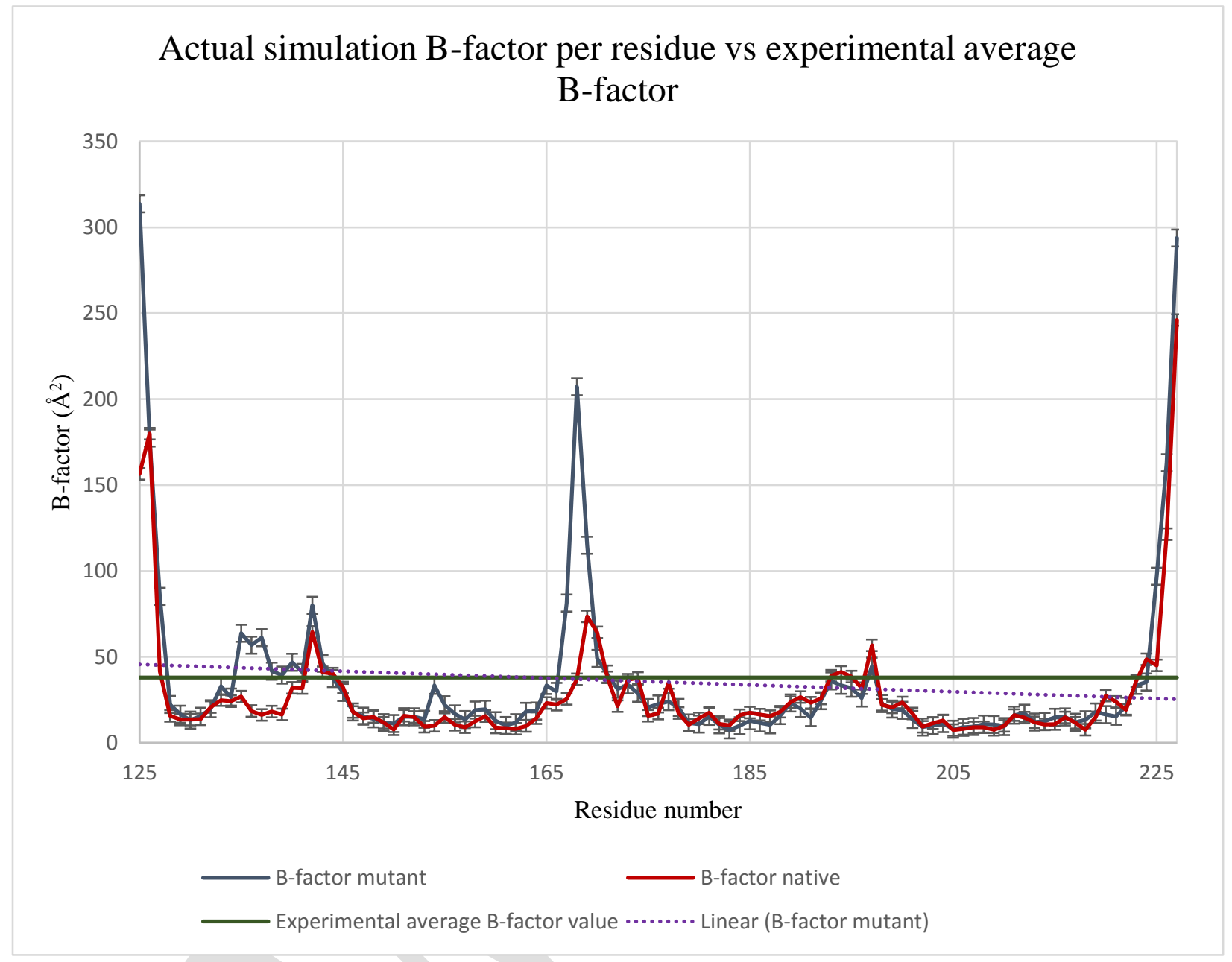

Fig. 8. The figure illustrates the calculated actual B-factor values from Molecular Dynamics simulations of both the native human $\mathrm{PrP}_{\text {nat }}$ prion and the FI-Prion with Met129 mutation in place of Val129 starting from the same crystal structure (PDB Code $=3 \mathrm{HAK}$ ). The average mutant Bfactor values converge to the average experimental B-factor value, whose respective X-Ray structure was obtained from the Protein Data Bank (PDB Code $=3 \mathrm{HES}$ ). It is evident that the FI- 
Prion protein (B-Factor mutant) displays more flexible regions between residues 126 to 144 , involving the mutation itself, compared to the native prion form (red). 


\section{Tables}

Table 1. The table shows a list of known prion mutations in humans and the corresponding diseases. $\mathrm{AD}=$ Alzheimer's Disease, $\mathrm{CJD}=$ Creutzfeldt-Jackob, PD = Parkinson's Disease, GSS = Gerstmann-Sträussler-Scheinker Syndrome, sFI = Sporadic Fatal Insomnia and FFI = Fatal Familial Insomnia (24-28).

\begin{tabular}{|l|l|l|}
\hline Codon & Mutation & Disease \\
\hline $\mathbf{1 0 2}$ & proline to leucine & GSS \\
\hline $\mathbf{1 0 5}$ & proline to leucine & GSS \\
\hline $\mathbf{1 1 7}$ & alanine to valine & GSS \\
\hline $\mathbf{1 9 8}$ & phenylalanine to serine & GSS \\
\hline $\mathbf{2 0 2}$ & aspartate to asparagine & GSS, PD \\
\hline $\mathbf{2 1 2}$ & glutamine to proline & GSS \\
\hline $\mathbf{2 1 7}$ & glutamine to arginine & GSS \\
\hline $\mathbf{2 1 8}$ & valine to alanine & GSS \\
\hline $\mathbf{1 2 9}$ & valine to methionine & sFI FFI, CJD, PD \\
\hline $\mathbf{1 7 8}$ & aspartate to asparagine & FFI, CJD \\
\hline $\mathbf{1 7 8}$ & aspartate to deletion & AD \\
\hline $\mathbf{1 4 8}$ & histidine to arginine & CJD \\
\hline $\mathbf{2 0 0}$ & glutamate to lysine & CJD \\
\hline $\mathbf{2 1 9}$ & Several & CJD \\
\hline $\mathbf{1 8 0}$ & valine to isoleucine & CJD \\
\hline $\mathbf{2 3 2}$ & methionine to arginine & AD, CJD, PD \\
\hline $\mathbf{1 2 7}$ & glycine to valine & Kuru \\
\hline $\mathbf{1 4 5}$ & tyrosine to nonsense & AD \\
\hline $\mathbf{1 6 3}$ & tyrosine to nonsense & AD \\
\hline $\mathbf{2 2 6}$ & tyrosine to nonsense & AD \\
\hline $\mathbf{2 2 7}$ & glutamine to nonsense & AD \\
\hline
\end{tabular}

\section{Supplementary Materials:}

Materials and Methods: The three-dimensional crystal structure of the human native prion protein was downloaded $(\mathrm{PDB}$ code $=3 \mathrm{HAK})$. The native structure was consequently mutated at codon 129, using NAMD (version 2.2 (C) obtaining the FI-Prion. The structures were 
analysed by using VMD. Both the native prion ( $\left.\mathrm{PrP}_{\text {nat }}\right)$ and the FI-Prion were then parameterised with the Charmm 27 force fields and the TIP3P explicit solvent model was used. The proteins were ultimately submerged in 20000 water molecules. The systems were then minimised by applying a convergence criterion of $0.01 \mathrm{kcal} / \mathrm{mol}$. Successively, a first equilibration was performed for the solvent for $10 \mathrm{ps}$ at $310 \mathrm{~K}$, using a Langevin thermostat, followed by $5 \mathrm{~ns}$ MD simulations in isothermal and isobaric conditions. A further MD simulation was carried out at a higher temperature $(364 \mathrm{~K})$ to investigate flexibility behaviour and stability under heating. All the simulations were executed with the optimal cut-off and with PME. Both simulations were also performed with PBC and RMS fluctuations (Figure 5) were obtained (29). The RMSF were then converted into $B-F A C T O R S$ by using the generic formula $B=\frac{8}{3} \pi^{2}(R M S F)^{2}(31-32)$. 
Copyright notice. The author is the legal and entitled owner of the property in reference and nobody else can legally take, use, claim ownership, republish, reprint, create or generate the content without his/her written and legal consent. Any discrimination or racial harassment by predatory reprint servers/publishers or by plagiarists is unlawful and therefore not the responsibility of the author, but of the courts and the current journal/preprint server to raise with the courts. (All rights reserved, 2016). 